\title{
THE COHOMOLOGY ALGEBRAS OF ORIENTABLE SEIFERT MANIFOLDS AND APPLICATIONS TO LUSTERNIK-SCHNIRELMANN CATEGORY
}

\author{
J. BRYDEN and P. ZVENGROWSKI \\ Department of Mathematics and Statistics, University of Calgary \\ Calgary, Alberta T2N 1N4, Canada \\ E-mail: bryden@acs.ucalgary.ca, zvengrow@acs.ucalgary.ca
}

\begin{abstract}
This note gives a complete description of the cohomology algebra of any orientable Seifert manifold with $\mathbb{Z} / p$ coefficients, for an arbitrary prime $p$. As an application, the existence of a degree one map from an orientable Seifert manifold onto a lens space is completely determined. A second application shows that the Lusternik-Schnirelmann category for a large class of Seifert manifolds is equal to 3 , which in turn is used to verify the Ganea conjecture for these Seifert manifolds.
\end{abstract}

1. Introduction. Throughout this paper $M$ will denote an orientable Seifert fibred manifold and $\tilde{M}$ its universal cover. Furthermore, $\Pi$ will denote the fundamental group $\pi_{1}(M)$ of $M$ and $R=\mathbb{Z} \Pi$ is its integral group ring. Then using the standard notation introduced in 1932 by Seifert [17], [18], either $M \cong\left(O, o, g \mid e:\left(a_{1}, b_{1}\right), \ldots,\left(a_{m}, b_{m}\right)\right)$ or $M \cong\left(O, n, g \mid e:\left(a_{1}, b_{1}\right), \ldots,\left(a_{m}, b_{m}\right)\right)$. In the first case $M$ is orientable with orientable orbit surface $V_{g}$ of genus $g \geq 0$, while in the second case $M$ is orientable with nonorientable orbit surface $W_{g}$ (a 2 -sphere with $g$ cross caps), $g \geq 1$. In both cases $e$ is the Euler number, $m$ is the number of singular fibres and, for each $i,\left(a_{i}, b_{i}\right)$ is a pair of relatively prime integers that characterize the twisting of the $i$-th singular fibre. For other references on Seifert manifolds see [8], [12], [13], [16].

The cohomology algebra of the orientable Seifert manifolds with orbit surface $S^{2}$ and $\mathbb{Z} / 2$ coefficients is described in [1]. The present paper provides a two-fold generalization of these results to the cohomology algebra of any orientable Seifert manifold with $\mathbb{Z} / p$ coefficients, for any prime $p$. The techniques used are similar to those formulated in [1] and [2]. We proceed by identifying the cohomology of the Seifert manifold with the

1991 Mathematics Subject Classification: 57M25, 20F38, 20J05, 55M30.

Key words and phrases: Seifert manifolds, cohomology algebra, diagonal map, cup products, degree one maps, Lusternik-Schnirelmann category, cup product length.

The paper is in final form and no version of it will be published elsewhere. 
group cohomology of its fundamental group, $\Pi$, which is assumed to be infinite. This can be done because such Seifert manifolds (apart from the two exceptions $\mathbb{R P}^{3} \# \mathbb{R} P^{3}$ and $\left.S^{1} \times S^{2}\right)$ are Eilenberg-MacLane spaces of type $K(\Pi, 1)$. This reduces the problem to a purely algebraic computation in group cohomology. The equivariant chain complex of the universal cover, $\tilde{M}$, of $M$ can then be used to compute $H^{*}(\Pi ; \mathbb{Z} / p)$. The calculation of the cup products can then be carried out from their definition by constructing a diagonal approximation for the equivariant chain complex. The assumption that $|\Pi|=\infty$ is not restrictive since there are relatively few Seifert manifolds with finite fundamental group and these are all well known. The construction of the equivariant chain complex, and the diagonal approximation, are stated in $\S 2$.

In $\S 3$, the diagonal approximation is used to calculate the $\mathbb{Z} / p$ cup products and the $\mathbb{Z} / p$ Bockstein maps. These results are then used in $\S 4$ to determine when an orientable Seifert manifold admits a degree one map onto a lens space $L(p, q)$, for a given prime $p$. The formulae and theorems in $\S 2$ and $\S 3$ are, for the most part, stated without proof. The complete details of the proofs of all of these theorems, which are somewhat lengthy and algebraically technical, will be given in [3].

The application to the Lusternik-Schnirelmann category (see Theorem 4.5) follows directly from the calculation of the cup products for the orientable Seifert manifolds and the well known result relating the cup product length to the Lusternik-Schnirelmann category. This theorem is proved in $\S 4$. The result on cup product length and the Künneth Theorem enable us to resolve the corresponding cases of the Ganea conjecture (cf. Theorem 4.6).

While $\S 2$ gives all the basic formulae necessary for the calculation of the cup products in $\S 3$ and the applications in $\S 4$, these formulae are algebraically complex. The reader, after looking at the notation and conventions in $\S 2$, may prefer to pass directly to the last two sections without examining the formulae $\left(R_{i . j}\right),\left(R_{i . j}^{*}\right)$ and $\Delta$ (Theorem 2.1) in detail, at least on first reading.

2. The equivariant chain complex and the diagonal approximation. In [2], the equivariant chain complex for the the universal cover $\tilde{M}$ was constructed in the genus zero case where the associated orbit surface is $S^{2}$, using results from [5] and [14]. In the general case, where the Seifert manifold is orientable and can have any orbit surface, this chain complex is modified using the same techniques given in [1] $\S 2,[2] \S 4$, and the resulting equivariant chain complex is now described in all possible cases.

(Oo): The Seifert manifold and the associated orbit surface are both orientable. Here

$$
\begin{aligned}
\Pi=\left\langle s_{1}, \ldots, s_{m}, v_{1}, w_{1}, \ldots, v_{g}, w_{g}, h\right| \\
\left.\quad\left[s_{j}, h\right], s_{j}^{a_{j}} h^{b_{j}},\left[v_{j}, h\right],\left[w_{j}, h\right], s_{1} \ldots s_{m}\left[v_{1}, w_{1}\right] \ldots\left[v_{g}, w_{g}\right] h^{-e}\right\rangle .
\end{aligned}
$$

Note that $h$ is a central element in $\Pi$.

The equivariant chain complex $\mathcal{C}$ for the universal cover $\tilde{M}$ is formed from the free $R$-modules in dimensions $0,1,2,3$ with free generators

$$
\begin{array}{lll}
\left(G_{0}\right) & 0: & \sigma_{0}^{0}, \ldots, \sigma_{m}^{0} ; \\
\left(G_{1}\right) & 1: & \sigma_{1}^{1}, \ldots, \sigma_{m}^{1} ; \rho_{0}^{1}, \ldots, \rho_{m}^{1} ; \nu_{1}^{1}, \omega_{1}^{1}, \ldots, \nu_{g}^{1}, \omega_{g}^{1} ; \eta_{0}^{1}, \ldots, \eta_{m}^{1}
\end{array}
$$




$$
2: \quad \sigma_{1}^{2}, \ldots, \sigma_{m}^{2} ; \rho_{0}^{2}, \ldots, \rho_{m}^{2} ; \nu_{1}^{2}, \omega_{1}^{2}, \ldots, \nu_{g}^{2}, \omega_{g}^{2} ; \mu_{0}^{2}, \ldots, \mu_{m}^{2} ; \delta^{2} ;
$$

$$
3: \sigma_{0}^{3}, \ldots, \sigma_{m}^{3} ; \delta^{3}
$$

The definition of the boundary map, $\partial$, of the chain complex $C$, requires the following conventions and definitions in the group ring $R$. First of all, in addition to the list of generators given in $\left(G_{1}\right),\left(G_{2}\right)$ adopt the notation $\sigma_{0}^{1}=0, \sigma_{0}^{2}=0$ and set $s_{0}=h^{-e}$. Next, let $r_{j}=s_{0} s_{1} \ldots s_{j}$ for $-1 \leq j \leq m,\left(r_{-1}=1\right)$ and $r_{m+j}:=r_{m} \prod_{k=1}^{j}\left[v_{k}, w_{k}\right]$ for $1 \leq j \leq g$. Observe that $r_{m+g}=1$. Given relatively prime integers $a_{j}>0, b_{j}>0$, choose integers $c_{j}>0, d_{j}>0$ so that $\left|\begin{array}{l}a_{j} \\ c_{j} b_{j}\end{array}\right|=1$ and let $t_{j}=s_{j}^{c_{j}} h^{d_{j}}$. Then $s_{j}=t_{j}^{-b_{j}}$ and $h=t_{j}^{a_{j}}$. When $j=0$ set $a_{0}=1, b_{0}=e$, giving $s_{0}=h^{-e}$, as before. Now define the Laurent polynomials

$$
\begin{aligned}
f_{l, j} & =1+t_{j}+\ldots+t_{j}^{l-1}, l \geq 1 ; \quad f_{a_{j}, j}=F_{j}=\frac{t_{j}^{a_{j}}-1}{t_{j}-1}, \\
g_{l, j} & =t_{j}^{-1}+t_{j}^{-2}+\ldots+t_{j}^{-l}, l \geq 1 ; \quad g_{b_{j}, j}=G_{j}=\frac{1-t_{j}^{-b_{j}}}{t_{j}-1}, \\
P_{j} & =1+t_{j}^{-b_{j}}+\ldots+t_{j}^{-b_{j}\left(c_{j}-1\right)}, \\
Q_{j} & =1+t_{j}^{a_{j}}+\ldots+t_{j}^{a_{j}\left(d_{j}-1\right)} .
\end{aligned}
$$

In particular, $F_{0}=1$ and $G_{0}=\left(1-h^{-e}\right) /(h-1)$. Finally, define the chains:

$$
\begin{aligned}
\pi_{j}^{1} & :=r_{j-1}\left(\sigma_{j}^{1}+\rho_{j}^{1}\right)-r_{j} \sigma_{j}^{1}, \\
\pi_{m+j}^{1} & :=r_{m+j-1}\left(1-v_{j} w_{j} v_{j}^{-1}\right) \nu_{j}^{1}+\left(r_{m+j-1} v_{j}-r_{m+j}\right) \omega_{j}^{1}, \\
\pi_{j}^{2} & :=-r_{j-1}\left(\sigma_{j}^{2}+\rho_{j}^{2}\right)+r_{j} \sigma_{j}^{2}, \\
\pi_{m+j}^{2} & :=r_{m+j-1}\left(v_{j} w_{j} v_{j}^{-1}-1\right) \nu_{j}^{2}+\left(r_{m+j}-r_{m+j-1} v_{j}\right) \omega_{j}^{2} .
\end{aligned}
$$

The free resolution $\mathcal{C}$ is given by the exact sequence

$$
\mathcal{C}: \quad 0 \longrightarrow C_{3} \stackrel{\partial_{3}}{\longrightarrow} C_{2} \stackrel{\partial_{2}}{\longrightarrow} C_{1} \stackrel{\partial_{1}}{\longrightarrow} C_{0} \stackrel{\varepsilon}{\longrightarrow} \mathbb{Z} \longrightarrow 0
$$

and the differentials are defined by

$$
\begin{array}{ll}
\left(R_{1,1}\right) & \partial \sigma_{j}^{1}=\sigma_{j}^{0}-\sigma_{0}^{0}, \quad 1 \leq j \leq m, \\
\left(R_{1,2}\right) & \partial \rho_{j}^{1}=\left(s_{j}-1\right) \sigma_{j}^{0}, \quad 0 \leq j \leq m, \\
\left(R_{1,3}\right) & \partial \eta_{j}^{1}=(h-1) \sigma_{j}^{0}, \quad 0 \leq j \leq m, \\
\left(R_{1,4}\right) & \partial \nu_{j}^{1}=\left(v_{j}-1\right) \sigma_{0}^{0}, \quad \partial \omega_{j}^{1}=\left(w_{j}-1\right) \sigma_{0}^{0}, \quad 1 \leq j \leq g, \\
\left(R_{2,1}\right) & \partial \sigma_{j}^{2}=\eta_{0}^{1}-\eta_{j}^{1}+(h-1) \sigma_{j}^{1}, \quad 1 \leq j \leq m, \\
\left(R_{2,2}\right) & \partial \rho_{j}^{2}=\left(1-s_{j}\right) \eta_{j}^{1}+(h-1) \rho_{j}^{1}, \quad 0 \leq j \leq m, \\
\left(R_{2,3}\right) & \partial \nu_{j}^{2}=\left(1-v_{j}\right) \eta_{0}^{1}+(h-1) \nu_{j}^{1}, \quad \partial \omega_{j}^{2}=\left(1-w_{j}\right) \eta_{0}^{1}+(h-1) \omega_{j}^{1}, \quad 1 \leq j \leq g, \\
\left(R_{2,4}\right) & \partial \delta^{2}=\sum_{j=0}^{m} \pi_{j}^{1}+\sum_{j=1}^{g} \pi_{m+j}^{1}, \\
\left(R_{2,5}\right) & \partial \mu_{j}^{2}=F_{j} \cdot \rho_{j}^{1}+G_{j} \cdot \eta_{j}^{1}, \quad 0 \leq j \leq m,
\end{array}
$$




$$
\begin{aligned}
& \left(R_{3,1}\right) \quad \partial \sigma_{j}^{3}=\rho_{j}^{2}+\left(1-t_{j}\right) \mu_{j}^{2}, \quad 0 \leq j \leq m, \\
& \left(R_{3,2}\right) \quad \partial \delta^{3}=(1-h) \delta^{2}-\sum_{j=0}^{m} \pi_{j}^{2}-\sum_{j=1}^{g} \pi_{m+j}^{2} .
\end{aligned}
$$

REMARK 2.1. Observe that $\pi_{0}^{1}=\rho_{0}^{1}, \pi_{0}^{2}=-\rho_{0}^{2}$, and

$$
\partial \pi_{j}^{1}=\left(r_{j}-r_{j-1}\right) \sigma_{0}^{0}, \quad \partial \pi_{m+j}^{2}=\left(r_{j}-r_{j-1}\right) \eta_{0}^{1}+(1-h) \pi_{m+j}^{1}, \quad 0 \leq j \leq m+g .
$$

(On): The Seifert manifold is orientable while the associated orbit surface is not orientable. In this case the cell complex of $\tilde{M}$ does not include the cells $\omega_{j}^{1}, \omega_{j}^{2}$ listed in $\left(G_{1}\right)$ and $\left(G_{2}\right)$ but otherwise has the same generators as the (Oo) case. The fundamental group has the following presentation:

$$
\Pi=\left\langle s_{1}, \ldots, s_{m}, v_{1}, \ldots, v_{g}, h \mid\left[s_{j}, h\right], s_{j}^{a_{j}} h^{b_{j}}, v_{j} h v_{j}^{-1} h, s_{1} \ldots s_{m} v_{1}^{2} \ldots v_{g}^{2} h^{-e}\right\rangle .
$$

Define the chains:

$$
\begin{aligned}
\pi_{m+j}^{1} & :=r_{m+j-1}\left(1+v_{j}\right) \nu_{j}^{1}, \\
\pi_{m+j}^{2} & :=r_{m+j-1}\left(h v_{j}-1\right) \nu_{j}^{2}, \text { where } \\
r_{m+j} & :=s_{o} \ldots s_{m} \prod_{k=1}^{j} v_{j}^{2} .
\end{aligned}
$$

Note that although $h$ is not central here, as in the $(O, o)$ case, it does commute with each $s_{j}, v_{i}^{2}$, and hence also with $r_{k}, 0 \leq k \leq m+g$. The boundary relations $\left(R_{i, j}\right)$ without $\nu_{j}, \omega_{j}, v_{j}, w_{j}$ are the same as in the case of the orientable surface. One has to replace the other $\left(R_{i, j}\right)$ by the following $\left(R_{i, j}^{*}\right)$.

$$
\begin{array}{ll}
\left(R_{1,4}^{*}\right) & \partial \nu_{j}^{1}=\left(v_{j}-1\right) \sigma_{0}^{0}, \quad 1 \leq j \leq g, \\
\left(R_{2,3}^{*}\right) . & \partial \nu_{j}^{2}=\left(1+h v_{j}\right) \eta_{0}^{1}+(h-1) \nu_{j}^{1}, \quad 1 \leq j \leq g .
\end{array}
$$

The free resolution $\mathcal{C}$, given above, suffices to find the additive structure of $H^{*}(M ; A)$. However, to find the ring structure (i.e. the cup products), make $\mathcal{C} \otimes \mathcal{C}$ into an $R$-chain complex by setting $\partial(x \otimes y)=\partial x \otimes y+(-1)^{\operatorname{deg}(x)} x \otimes \partial y$, and $(n u+m v)(x \otimes y)=$ $n(u x \otimes u y)+m(v x \otimes v y)$ for $m, n \in \mathbb{Z}, u, v \in \Pi, x, y \in \mathcal{C}$. Then seek a diagonal approximation $\Delta: \mathcal{C} \rightarrow \mathcal{C} \otimes \mathcal{C}$ such that

(a) $\Delta$ is an $R$-chain map,

(b) $\Delta$ preserves augmentation, that is, there is a commutative diagram:

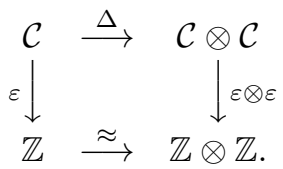

Such a diagonal map $\Delta$ must exist by the theorem of acyclic models (cf. Steenrod and Epstein [21] Chapter 5), but it must be found explicitly. One of the main difficulties in the construction of this diagonal map is that there are a variety of choices at each stage of the construction. Of course, by (a), it suffices to define $\Delta$ on the (free) generators of the complex $\mathcal{C}$.

Before stating the central result, we introduce the 1-chain $\tau_{j}^{1}=P_{j} \rho_{j}^{1}+t_{j}^{-b_{j} c_{j}} Q_{j} \eta_{j}^{1}$. Also, for convenience let $\bar{m}=m-1$. 
Diagonal Approximation Theorem 2.2. The diagonal approximation of the equivariant chain complex $\mathcal{C}$ is defined on the generators of $\mathcal{C}$ as follows:

$(\mathrm{Oo}): M \cong\left(O, o ; g \mid e:\left(a_{1}, b_{1}\right), \ldots,\left(a_{m}, b_{m}\right)\right)$.

$$
\begin{aligned}
& \Delta\left(\sigma_{j}^{0}\right)=\sigma_{j}^{0} \otimes \sigma_{j}^{0}, \quad \Delta\left(\sigma_{j}^{1}\right)=\sigma_{j}^{1} \otimes \sigma_{j}^{0}+\sigma_{0}^{0} \otimes \sigma_{j}^{1}, \\
& \Delta\left(\rho_{j}^{1}\right)=s_{j} \sigma_{j}^{0} \otimes \rho_{j}^{1}+\rho_{j}^{1} \otimes \sigma_{j}^{0}, \quad \Delta\left(\eta_{j}^{1}\right)=h \sigma_{j}^{0} \otimes \eta_{j}^{1}+\eta_{j}^{1} \otimes \sigma_{j}^{0}, \\
& \Delta\left(\nu_{j}^{1}\right)=\nu_{j}^{1} \otimes \sigma_{0}^{0}+v_{j} \sigma_{0}^{0} \otimes \nu_{j}^{1}, \quad \Delta\left(\omega_{j}^{1}\right)=\omega_{j}^{1} \otimes \sigma_{0}^{0}+w_{j} \sigma_{0}^{0} \otimes \omega_{j}^{1}, \\
& \Delta\left(\sigma_{j}^{2}\right)=h \sigma_{0}^{0} \otimes \sigma_{j}^{2}-h \sigma_{j}^{1} \otimes \eta_{j}^{1}+\sigma_{j}^{2} \otimes \sigma_{j}^{0}+\eta_{0}^{1} \otimes \sigma_{j}^{1}, \\
& \Delta\left(\rho_{j}^{2}\right)=\rho_{j}^{2} \otimes \sigma_{j}^{0}+s_{j} \eta_{j}^{1} \otimes \rho_{j}^{1}-h \rho_{j}^{1} \otimes \eta_{j}^{1}+h s_{j} \sigma_{j}^{0} \otimes \rho_{j}^{2}, \\
& \Delta\left(\nu_{j}^{2}\right)=\nu_{j}^{2} \otimes \sigma_{0}^{0}+v_{j} \eta_{0}^{1} \otimes \nu_{j}^{1}-h \nu_{j}^{1} \otimes \eta_{0}^{1}+h v_{j} \sigma_{0}^{0} \otimes \nu_{j}^{2}, \\
& \Delta\left(\omega_{j}^{2}\right)=\omega_{j}^{2} \otimes \sigma_{0}^{0}+w_{j} \eta_{0}^{1} \otimes \omega_{j}^{1}-h \omega_{j}^{1} \otimes \eta_{0}^{1}+h w_{j} \sigma_{0}^{0} \otimes \omega_{j}^{2}, \\
& \Delta\left(\mu_{j}^{2}\right)=\mu_{j}^{2} \otimes t_{j}^{-b_{j}} \sigma_{j}^{0}+t_{j}^{a_{j}-b_{j}} \sigma_{j}^{0} \otimes \mu_{j}^{2}-\sum_{k=0}^{a_{j}-1} \sum_{l=-b_{j}}^{k-1} t_{j}^{k} \rho_{j}^{1} \otimes t_{j}^{l} \tau_{j}^{1}-\sum_{l=0}^{a_{j}-1} \sum_{k=l-b_{j}}^{a_{j}-b_{j}-1} t_{j}^{k} \tau_{j}^{1} \otimes t_{j}^{l} \rho_{j}^{1} \\
& -\sum_{k=1-b_{j}}^{-1} \sum_{l=-b_{j}}^{k-1} t_{j}^{k} \eta_{j}^{1} \otimes t_{j}^{l} \tau_{j}^{1}+\sum_{l=1-b_{j}}^{-1} \sum_{k=a_{j}-b_{j}}^{a_{j}+l-1} t_{j}^{k} \tau_{j}^{1} \otimes t_{j}^{l} \eta_{j}^{1}-G_{j} \sum_{r=1}^{a_{j}-1} t_{j}^{r} \tau_{j}^{1} \otimes f_{r, j} \tau_{j}^{1} \\
& -F_{j} \sum_{r=1}^{b_{j}} t_{j}^{-r} \tau_{j}^{1} \otimes g_{r, j} \tau_{j}^{1} \\
& \Delta\left(\delta^{2}\right)=A+B,
\end{aligned}
$$

where

$$
\begin{aligned}
A= & \delta^{2} \otimes s_{0} \sigma_{0}^{0}+r_{m-1} \sigma_{0}^{0} \otimes \delta^{2}+\pi_{m}^{1} \otimes \pi_{m}^{1}+\rho_{0}^{1} \otimes \rho_{0}^{1}+\pi_{m}^{1} \otimes \rho_{0}^{1} \\
& -\sum_{j=2}^{m-1} \sum_{i=1}^{j-1} \pi_{j}^{1} \otimes \pi_{i}^{1}-\sum_{j=1}^{m} \pi_{j}^{1} \otimes r_{j-1} \sigma_{j}^{1}+\sum_{j=1}^{m} r_{j} \sigma_{j}^{1} \otimes r_{j-1} \rho_{j}^{1},
\end{aligned}
$$

and

$$
\begin{aligned}
B= & \sum_{j=1}^{g} \pi_{m+j}^{1} \otimes \rho_{0}^{1}+\sum_{j=1}^{g} \sum_{k=0}^{j-1} \pi_{m+k}^{1} \otimes \pi_{m+j}^{1}+\sum_{j=1}^{g} r_{\bar{m}+j} \nu_{j}^{1} \otimes \pi_{m+j}^{1} \\
& +\sum_{j=1}^{g} r_{\bar{m}+j} v_{j} \omega_{j}^{1} \otimes \pi_{m+j}^{1}-\sum_{j=1}^{g} r_{\bar{m}+j} v_{j} \omega_{j}^{1} \otimes r_{\bar{m}+j} \nu_{j}^{1} \\
& +\sum_{j=1}^{g} r_{\bar{m}+j} v_{j} w_{j} v_{j}^{-1} \nu_{j}^{1} \otimes r_{m+j} \omega_{j}^{1}, \\
\Delta\left(\sigma_{j}^{3}\right)=\sigma_{j}^{3} \otimes & \sigma_{j}^{0}+t_{j}^{a_{j}-b_{j}} \sigma_{j}^{0} \otimes \sigma_{j}^{3}-t_{j} \mu^{2} \otimes t_{j}^{-b_{j}} \tau_{j}^{1}-t_{j}^{a_{j}-b_{j}} \tau_{j}^{1} \otimes t_{j} \mu_{j}^{2}+t_{j} \mu_{j}^{2} \otimes G_{j} \tau_{j}^{1} \\
- & \mu_{j}^{2} \otimes G_{j} \tau_{j}^{1}-t_{j}^{-b_{j}} P_{j} \mu_{j}^{2} \otimes\left(\rho_{j}^{1}+G_{j} \tau_{j}^{1}\right)-t_{j}^{a_{j}}\left(\rho_{j}^{1}+G_{j} \tau_{j}^{1}\right) \otimes P_{j} \mu_{j}^{2},
\end{aligned}
$$




$$
\Delta\left(\delta^{3}\right)=A^{\prime}+B^{\prime},
$$

where

$$
\begin{aligned}
A^{\prime}= & \delta^{3} \otimes s_{0} \sigma_{0}^{0}+h r_{m-1} \sigma_{0}^{0} \otimes \delta^{3}-h \delta^{2} \otimes s_{0} \eta_{0}^{1}-r_{m-1} \eta_{0}^{1} \otimes \delta^{2}-\rho_{0}^{2} \otimes \rho_{0}^{1}+h \rho_{0}^{1} \otimes \rho_{0}^{2} \\
& +\pi_{m}^{2} \otimes \pi_{m}^{1}-h \pi_{m}^{1} \otimes \pi_{m}^{2}+\pi_{m}^{2} \otimes \rho_{0}^{1}+h \pi_{m}^{1} \otimes \rho_{0}^{2}-\sum_{j=2}^{m-1} \sum_{i=1}^{j-1} \pi_{j}^{2} \otimes \pi_{i}^{1} \\
& +\sum_{j=2}^{m-1} \sum_{i=1}^{j-1} h \pi_{j}^{1} \otimes \pi_{i}^{2}-\sum_{j=1}^{m} \pi_{j}^{2} \otimes r_{j-1} \sigma_{j}^{1}-\sum_{j=1}^{m} h \pi_{j}^{1} \otimes r_{j-1} \sigma_{j}^{2} \\
& -\sum_{j=1}^{m} r_{j} \sigma_{j}^{2} \otimes r_{j-1} \rho_{j}^{1}+\sum_{j=1}^{m} h r_{j} \sigma_{j}^{1} \otimes r_{j-1} \rho_{j}^{2},
\end{aligned}
$$

and

$$
\begin{aligned}
B^{\prime}= & \sum_{j=1}^{g} \pi_{m+j}^{2} \otimes \rho_{0}^{1}+\sum_{j=1}^{g} \sum_{k=0}^{j-1} \pi_{m+k}^{2} \otimes \pi_{m+j}^{1}-\sum_{j=1}^{g} \sum_{k=0}^{j-1} h \pi_{m+k}^{1} \otimes \pi_{m+j}^{2} \\
& -\sum_{j=1}^{g} r_{\bar{m}+j} \nu_{j}^{2} \otimes \pi_{m+j}^{1}-\sum_{j=1}^{g} h r_{\bar{m}+j} \nu_{j}^{1} \otimes \pi_{m+j}^{2}-\sum_{j=1}^{g} h r_{\bar{m}+j} v_{j} \omega_{j}^{1} \otimes \pi_{m+j}^{2} \\
& -\sum_{j=1}^{g} h r_{\bar{m}+j} v_{j} \omega_{j}^{1} \otimes r_{\bar{m}+j} \nu_{j}^{2}+\sum_{j=1}^{g} r_{\bar{m}+j} v_{j} \omega_{j}^{2} \otimes r_{\bar{m}+j} \nu_{j}^{1}-\sum_{j=1}^{g} r_{\bar{m}+j} v_{j} \omega_{j}^{2} \otimes \pi_{m+j}^{1} \\
& -\sum_{j=1}^{g} r_{\bar{m}+j} v_{j} w_{j} v_{j}^{-1} \nu_{j}^{2} \otimes r_{m+j} \omega_{j}^{1}+\sum_{j=1}^{g} h r_{\bar{m}+j} v_{j} w_{j} v_{j}^{-1} \nu_{j}^{1} \otimes r_{m+j} \omega_{j}^{2} \\
& +\sum_{j=1}^{g} h \pi_{m+j}^{1} \otimes \rho_{0}^{2} .
\end{aligned}
$$

This completes the construction of the diagonal approximation for the case when both the Seifert manifold and the orbit surface are orientable.

(On): $M \cong\left(O, n ; g \mid e:\left(a_{1}, b_{1}\right), \ldots,\left(a_{m}, b_{m}\right)\right)$. In this case the diagonal on the equivariant chain complex $\mathcal{C}$ is defined as in the $($ Oo $)$ case, except on the generators $\omega_{j}^{1}, \omega_{j}^{2}$, which do not occur in this case, and on the generators $\nu_{j}^{2}, \delta^{2}, \delta^{3}$. On these generators, the diagonal is given by:

$$
\begin{aligned}
& \Delta\left(\nu_{j}^{2}\right)=\nu_{j}^{2} \otimes \sigma_{0}^{0}-h v_{j} \eta_{0}^{1} \otimes \nu_{j}^{1}-h \nu_{j}^{1} \otimes \eta_{0}^{1}+h v_{j} \sigma_{0}^{0} \otimes \nu_{j}^{2}+h v_{j} \eta_{0}^{1} \otimes h v_{j} \eta_{0}^{1}, \\
& \Delta\left(\delta^{2}\right)=A+B,
\end{aligned}
$$

where

$$
\begin{aligned}
A= & \delta^{2} \otimes s_{0} \sigma_{0}^{0}+r_{m-1} \sigma_{0}^{0} \otimes \delta^{2}+\pi_{m}^{1} \otimes \pi_{m}^{1}+\rho_{0}^{1} \otimes \rho_{0}^{1}+\pi_{m}^{1} \otimes \rho_{0}^{1} \\
& -\sum_{j=2}^{m-1} \sum_{i=1}^{j-1} \pi_{j}^{1} \otimes \pi_{i}^{1}-\sum_{j=1}^{m} \pi_{j}^{1} \otimes r_{j-1} \sigma_{j}^{1}+\sum_{j=1}^{m} r_{j} \sigma_{j}^{1} \otimes r_{j-1} \rho_{j}^{1},
\end{aligned}
$$


and

$$
\begin{aligned}
B= & \sum_{j=1}^{g} \pi_{m+j}^{1} \otimes \rho_{0}^{1}+\sum_{j=1}^{g} \sum_{k=0}^{j-1} \pi_{m+k}^{1} \otimes \pi_{m+j}^{1}+\sum_{j=1}^{g} r_{\bar{m}+j} \nu_{j}^{1} \otimes \pi_{m+j}^{1} \\
& +\sum_{j=1}^{g} r_{\bar{m}+j} v_{j} \nu_{j}^{1} \otimes r_{\bar{m}+j} v_{j} \nu_{j}^{1}, \\
\Delta\left(\delta^{3}\right)= & A^{\prime}+B^{\prime}
\end{aligned}
$$

where

$$
\begin{aligned}
A^{\prime}= & \delta^{3} \otimes s_{0} \sigma_{0}^{0}+h r_{m-1} \sigma_{0}^{0} \otimes \delta^{3}-h \delta^{2} \otimes s_{0} \eta_{0}^{1}-r_{m-1} \eta_{0}^{1} \otimes \delta^{2}-\rho_{0}^{2} \otimes \rho_{0}^{1}+h \rho_{0}^{1} \otimes \rho_{0}^{2} \\
& +\pi_{m}^{2} \otimes \pi_{m}^{1}-h \pi_{m}^{1} \otimes \pi_{m}^{2}+\pi_{m}^{2} \otimes \rho_{0}^{1}+h \pi_{m}^{1} \otimes \rho_{0}^{2}-\sum_{j=2}^{m-1} \sum_{i=1}^{j-1} \pi_{j}^{2} \otimes \pi_{i}^{1} \\
& +\sum_{j=2}^{m-1} \sum_{i=1}^{j-1} h \pi_{j}^{1} \otimes \pi_{i}^{2}-\sum_{j=1}^{m} \pi_{j}^{2} \otimes r_{j-1} \sigma_{j}^{1}-\sum_{j=1}^{m} h \pi_{j}^{1} \otimes r_{j-1} \sigma_{j}^{2} \\
& -\sum_{j=1}^{m} r_{j} \sigma_{j}^{2} \otimes r_{j-1} \rho_{j}^{1}+\sum_{j=1}^{m} h r_{j} \sigma_{j}^{1} \otimes r_{j-1} \rho_{j}^{2},
\end{aligned}
$$

and

$$
\begin{aligned}
B^{\prime}= & \sum_{j=1}^{g} \pi_{m+j}^{2} \otimes \rho_{0}^{1}+\sum_{j=1}^{g} \sum_{k=0}^{j-1} \pi_{m+k}^{2} \otimes \pi_{m+j}^{1}-\sum_{j=1}^{g} \sum_{k=0}^{j-1} h \pi_{m+k}^{1} \otimes \pi_{m+j}^{2} \\
& -\sum_{j=1}^{g} r_{\bar{m}+j} \nu_{j}^{2} \otimes \pi_{m+j}^{1}-\sum_{j=1}^{g} h r_{\bar{m}+j} \nu_{j}^{1} \otimes \pi_{m+j}^{2}+\sum_{j=1}^{g} h r_{\bar{m}+j} v_{j} \nu_{j}^{2} \otimes r_{\bar{m}+j} v_{j} \nu_{j}^{1} \\
& -\sum_{j=1}^{g} h r_{\bar{m}+j} v_{j} \nu_{j}^{1} \otimes h r_{\bar{m}+j} v_{j} \nu_{j}^{2}+\sum_{j=1}^{g} h r_{\bar{m}+j} v_{j} \nu_{j}^{2} \otimes h r_{\bar{m}+j} v_{j} \eta_{0}^{1} \\
& +\sum_{j=1}^{g} r_{m+j} \eta_{0}^{1} \otimes h r_{\bar{m}+j} v_{j} \nu_{j}^{2}+\sum_{j=1}^{g} h \pi_{m+j}^{1} \otimes \rho_{0}^{2} .
\end{aligned}
$$

3. The cohomology algebra. The cohomology groups of the Seifert manifolds $M \cong\left(O, o ; g \mid e:\left(a_{1}, b_{1}\right), \ldots,\left(a_{m}, b_{m}\right)\right)$ and $M \cong\left(O, n ; g \mid e:\left(a_{1}, b_{1}\right), \ldots,\left(a_{m}, b_{m}\right)\right)$ are well known and can be determined by first calculating their homology from a $\mathrm{CW}$ decomposition (or by abelianizing $\pi_{1}(M)$ ) and then applying Poincaré duality. For our purposes it is most convenient to calculate them directly from the cochain complex $\operatorname{Hom}_{R}(\mathcal{C}, \mathbb{Z} / p)$, where $\mathbb{Z} / p$ is the trivial $R$-module. Specifically,

$$
H^{*}\left(\operatorname{Hom}_{R}(\mathcal{C}, \mathbb{Z} / p)\right) \cong H^{*}(\Pi ; \mathbb{Z} / p) \cong H^{*}(M ; \mathbb{Z} / p),
$$

since $M$ is a $K(\Pi, 1)$ space (cf. [11] Theorem 11.5). Any description of the cohomology algebra of a space, such as Theorems 3.1-3.7 below, is imprecise unless the generators of the various classes involved are also specified. The description of these generators 
is given in the Appendix in terms of specific cocycles in $\operatorname{Hom}_{R}(\mathcal{C}, \mathbb{Z} / p)$, and can be referred to when necessary. The proofs of all the results in this section are actually quite straightforward and follow directly from the results in $\S 2$. They are, however, omitted here (full details are given in [3]).

In order to describe the cohomology of these manifolds the following notational conventions are made. For any prime $p$, assume without loss of generality that $a_{1}, \ldots, a_{n_{p}} \equiv 0$ $(\bmod p)$ and $a_{n_{p}+1}, \ldots, a_{m} \neq \equiv 0(\bmod p)$. In this case there exist integers $a_{1}^{\prime}, \ldots, a_{n_{p}}^{\prime}$, such that $a_{1}=p a_{1}^{\prime}, \ldots, a_{n_{p}}=p a_{n_{p}}^{\prime}$. Choose integers $c_{i}, d_{i}$ so that $a_{i} d_{i}-b_{i} c_{i}=1$. Then, for $1 \leq i \leq n_{p}, b_{i}, c_{i} \neq \equiv 0(\bmod p)$. When $n_{p}=0$, that is when $a_{i} \not \equiv 0(\bmod p)$ for all $1 \leq i \leq m$, let $b_{1}, \ldots, b_{r} \equiv 0(\bmod p), b_{r+1}, \ldots, b_{m} \not \equiv 0(\bmod p)$. Then there exist $b_{1}^{\prime}, \ldots, b_{r}^{\prime}$ such that $b_{1}=p b_{1}^{\prime}, \ldots, b_{r}=p b_{r}^{\prime}$. Let $A=\prod_{i=1}^{m} a_{i}, A_{i}=a_{i}^{-1} A \in \mathbb{Z}$ and $C=\sum b_{i} A_{i}$. Observe that $A \not \equiv 0(\bmod p)$. Finally note that the same symbol will be used to denote an integer $s$ and its mod $p$ reduction. The context will remove any ambiguity.

The calculation of these cohomology groups gives the following results:

For $M \cong\left(O, o ; g \mid e:\left(a_{1}, b_{1}\right), \ldots,\left(a_{m}, b_{m}\right)\right)$,

(i) If $n_{p}>0$ then $H^{1}(M ; \mathbb{Z} / p) \approx H^{2}(M ; \mathbb{Z} / p) \approx(\mathbb{Z} / p)^{2 g+n_{p}-1}$.

(ii) If $n_{p}=0$, then
(a) $H^{1}(M ; \mathbb{Z} / p) \approx H^{2}(M ; \mathbb{Z} / p) \approx(\mathbb{Z} / p)^{2 g}$
if $A e+C \not \equiv 0(\bmod p)$ and
(b) $H^{1}(M ; \mathbb{Z} / p) \approx H^{2}(M ; \mathbb{Z} / p) \approx(\mathbb{Z} / p)^{2 g+1}$,
if $A e+C \equiv 0(\bmod p)$.

In the case when $M \cong\left(O, n ; g \mid e:\left(a_{1}, b_{1}\right), \ldots,\left(a_{m}, b_{m}\right)\right)$ then similarly

(i) If $n_{p}>0$ then $H^{1}(M ; \mathbb{Z} / p) \approx H^{2}(M ; \mathbb{Z} / p) \approx(\mathbb{Z} / p)^{g+n_{p}-1}$.

(ii) If $n_{p}=0$, then
(a) $H^{1}(M ; \mathbb{Z} / p) \approx H^{2}(M ; \mathbb{Z} / p) \approx(\mathbb{Z} / p)^{g}$,
if $A e+C \not \equiv 0(\bmod p)$ and
(b) $H^{1}(M ; \mathbb{Z} / p) \approx H^{2}(M ; \mathbb{Z} / p) \approx(\mathbb{Z} / p)^{g+1}$,
if $A e+C \equiv 0(\bmod p)$.

The remainder of this section will be devoted to the description of the cup products. Again we remind the reader that the generating classes in cohomology are described in the Appendix.

Theorem 3.1. Let $M \cong\left(O, o ; g \mid e:\left(a_{1}, b_{1}\right), \ldots,\left(a_{m}, b_{m}\right)\right)$ and let $\delta_{j k}$ denote the Kronecker delta.

If $n_{p}>0$, then as a graded vector space,

$$
H^{*}(M ; \mathbb{Z} / p)=\mathbb{Z} / p\left\{1, \alpha_{i}, \theta_{l}, \theta_{l}^{\prime}, \beta_{i}, \varphi_{l}, \varphi_{l}^{\prime}, \gamma \mid 2 \leq i \leq n_{p}, 1 \leq l \leq g\right\} .
$$

with $\alpha_{i}, \theta_{l}, \theta_{l}^{\prime}$ in degree $1, \beta_{i}, \varphi_{l}, \varphi_{l}^{\prime}$ in degree 2 , and $\gamma$ in degree 3 . Now set $\beta_{1}=$ $-\sum_{i=2}^{n} \beta_{i}$. Then, the non-trivial cup products in $H^{*}(M ; \mathbb{Z} / p)$ are given by:

(i) For $p=2$, let $2 \leq i, j \leq n_{2}$. Then

$$
\alpha_{i} \cdot \alpha_{j}=\left(\begin{array}{c}
a_{1} \\
2
\end{array}\right) \beta_{1}+\delta_{i j}\left(\begin{array}{c}
a_{i} \\
2
\end{array}\right) \beta_{i} .
$$

Furthermore, if $2 \leq k \leq n_{2}$ as well, then

$$
\alpha_{i} \cdot \alpha_{j} \cdot \alpha_{k}=\left(\begin{array}{c}
a_{1} \\
2
\end{array}\right) \gamma \text { if } i \neq j \text { or } j \neq k, \quad \alpha_{i}^{3}=\left[\left(\begin{array}{c}
a_{1} \\
2
\end{array}\right)+\left(\begin{array}{c}
a_{i} \\
2
\end{array}\right)\right] \gamma
$$


(ii) For any prime $p$, let $2 \leq i \leq n_{p}$ and $1 \leq l \leq g$. Then,

$$
\alpha_{i} \cdot \beta_{i}=-\gamma, \quad \theta_{l} \cdot \varphi_{l}^{\prime}=\theta_{l}^{\prime} \cdot \varphi_{l}=\gamma .
$$

Additionally, the mod $p$ Bockstein, $B_{p}$, on $H^{1}(M ; \mathbb{Z} / p)$ is given by

$$
\begin{aligned}
B_{p}\left(\alpha_{i}\right) & =-a_{i}^{\prime} c_{i} \beta_{i}+a_{1}{ }^{\prime} c_{1} \beta_{1} \in H^{2}(M ; \mathbb{Z} / p), \\
B_{p}\left(\theta_{l}\right) & =B_{p}\left(\theta_{l}^{\prime}\right)=0 .
\end{aligned}
$$

REMARKS 3.2. (i) For $p=2, B_{2}\left(\alpha_{i}\right)=S q^{1}\left(\alpha_{i}\right)=\alpha_{i}^{2}$. Thus the formula given in (i) for $\alpha_{i}^{2}$ agrees with the formula for $B_{2}\left(\alpha_{i}\right)$ given in (ii).

(ii) In case $n_{p}=1$, there are no $\alpha_{i}$ or $\beta_{i}$ classes. Furthermore, if $g=0$ there are no $\theta_{l}, \theta_{l}^{\prime}, \phi_{l}$, or $\phi_{l}^{\prime}$ classes.

(iii) Since $n_{p}>0$ only when there exists an $a_{i}$ that is divisible by $p$, it follows that $n_{p}=0$ for all but a finite number of primes $p$.

TheOREM 3.3. Let $M \cong\left(O, o ; g \mid e:\left(a_{1}, b_{1}\right), \ldots,\left(a_{m}, b_{m}\right)\right)$ and suppose that for some prime $p \geq 2, n_{p}=0$. In case $A e+C \not \equiv 0(\bmod p) H^{*}(M ; \mathbb{Z} / p)$ has generators $\theta_{l}, \theta_{l}^{\prime}, \varphi_{l}$, $\varphi_{l}^{\prime}, 1 \leq l \leq g$. If $A e+C \equiv 0(\bmod p)$, then

$$
H^{*}(M ; \mathbb{Z} / p)=\mathbb{Z} / p\left\{1, \alpha, \theta_{l}, \theta_{l}^{\prime}, \beta, \varphi_{l}, \varphi_{l}^{\prime}, \gamma \mid 1 \leq l \leq g\right\},
$$

where $\operatorname{deg}(\alpha)=1$ and $\operatorname{deg}(\beta)=2$.

The non-trivial cup products are given by:

(i) For $p=2$, if $A e+C \equiv 0(\bmod 2)$

$$
\alpha^{2}=\left[q+\frac{1}{2}(A e+C)\right] \beta,
$$

where $q$ is defined to be the number of $b_{i}, 1 \leq i \leq r$, which are congruent to 2 $(\bmod 4)$.

(ii) If $A e+C \equiv 0(\bmod p)$, for any $p$, then for $1 \leq l \leq g$,

$$
\begin{aligned}
\alpha \cdot \theta_{l} & =\varphi_{l}, & \alpha \cdot \theta_{l}^{\prime} & =\varphi_{l}^{\prime}, \\
\theta_{l} \cdot \theta_{l}^{\prime} & =\beta, & \alpha \cdot \beta & =-\gamma .
\end{aligned}
$$

(iii) In either case, for $1 \leq m \leq g$ (and for any $p$ ),

$$
\theta_{l} \cdot \varphi_{l}^{\prime}=\theta_{l}^{\prime} \cdot \varphi_{l}=\gamma
$$

Furthermore, the $\bmod p$ Bockstein on $H^{1}(M ; \mathbb{Z} / p)$ is given by:

$$
\begin{aligned}
& B_{p}(\alpha)=-A^{-1}\left[\sum_{i=1}^{r} b_{i}^{\prime} A_{i}+\frac{A e+C}{p}\right] \beta \in H^{2}(M ; \mathbb{Z} / p), \\
& B_{p}\left(\theta_{l}\right)=B_{p}\left(\theta_{l}^{\prime}\right)=0 .
\end{aligned}
$$

In the case when the Seifert manifold has a non-orientable orbit surface homeomorphic to $V_{g}=\mathbb{R P}^{2} \# \ldots \# \mathbb{R P}^{2}$, the cohomology algebra is now given.

TheOREM 3.4. Let $M \cong\left(O, n ; g \mid e:\left(a_{1}, b_{1}\right), \ldots,\left(a_{m}, b_{m}\right)\right)$. If for any prime $p$, $n_{p}>0$, then as a graded vector space,

$$
H^{*}(M ; \mathbb{Z} / p)=\mathbb{Z} / p\left\{1, \alpha_{i}, \theta_{l}, \beta_{i}, \varphi_{l}, \gamma \mid 2 \leq i \leq n, 1 \leq l \leq g\right\} .
$$


In this case let $\beta_{1}=-\sum_{i=2}^{n} \beta_{i}-2 \sum_{l=1}^{g} \varphi_{l}$. The non-trivial cup products in $H^{*}(M ; \mathbb{Z} / p)$ are given by:

(i) For $p=2$ and $2 \leq i, j \leq n_{2}$,

$$
\alpha_{i} \cdot \alpha_{j}=\left(\begin{array}{c}
a_{1} \\
2
\end{array}\right) \beta_{1}+\delta_{i j}\left(\begin{array}{c}
a_{i} \\
2
\end{array}\right) \beta_{i} .
$$

Moreover, if $2 \leq k \leq n_{2}$ as well, then

$$
\alpha_{i} \cdot \alpha_{j} \cdot \alpha_{k}=\left(\begin{array}{c}
a_{1} \\
2
\end{array}\right) \gamma \text { if } i \neq j \text { or } j \neq k, \quad \alpha_{i}^{3}=\left[\left(\begin{array}{c}
a_{1} \\
2
\end{array}\right)+\left(\begin{array}{c}
a_{i} \\
2
\end{array}\right)\right] \gamma
$$

(ii) For any prime $p$, let $2 \leq i, j \leq n_{p}$ and $1 \leq l \leq g$. Then,

$$
\alpha_{j} \cdot \beta_{j}=-\gamma \quad \text { and } \quad \theta_{l} \cdot \varphi_{l}=-\gamma \text {. }
$$

In addition, the mod $p$ Bockstein on $H^{1}(M, \mathbb{Z} / p)$ is given by:

$$
\begin{aligned}
B_{p}\left(\alpha_{j}\right) & =-a_{j}^{\prime} c_{j} \beta_{j}+a_{1}^{\prime} c_{1} \beta_{1}, \\
B_{p}\left(\theta_{l}\right) & =-2 a_{1}^{\prime} c_{1} \beta_{1} .
\end{aligned}
$$

REMARK 3.5. (i) When $p=2, B_{p}\left(\alpha_{i}\right)=\alpha_{i}^{2}$ as in Remark 3.2 (i) and the formulas given in Theorem 3.4 (i) and (ii) agree.

(ii) When $n_{p}=1$, there are no $\alpha_{i}$ or $\beta_{i}$ classes.

(iii) Note that in the (On) case $g \geq 1$ always holds.

Theorem 3.6. Let $M \cong\left(O, n ; g \mid e:\left(a_{1}, b_{1}\right), \ldots,\left(a_{m}, b_{m}\right)\right)$. Suppose, for some $p>2$, $n_{p}=0$. Then as a graded vector space

$$
H^{*}(M ; \mathbb{Z} / p)=\mathbb{Z} / p\left\{1, \theta_{l}, \varphi_{l}, \gamma \mid 1 \leq l \leq g\right\}
$$

In this case

$$
\theta_{l} \cdot \varphi_{l}=-\gamma, \text { for } 1 \leq l \leq g, \quad B_{p}\left(\theta_{l}\right)=0,
$$

while all other cup products are zero.

TheOrem 3.7. Let $M \cong\left(O, n ; g \mid e:\left(a_{1}, b_{1}\right), \ldots,\left(a_{m}, b_{m}\right)\right)$. Suppose that $p=2$ and $n_{2}=0$. If $A e+C \not \equiv 0(\bmod 2)$, then $H^{1}(M ; \mathbb{Z} / 2)$ is generated by the elements $\theta_{1}, \ldots, \theta_{g}$, and $H^{2}(M ; \mathbb{Z} / 2)$ has generators $\varphi_{1}, \ldots, \varphi_{g}$. In case $A e+C \equiv 0(\bmod 2)$ there are two additional generators $\alpha \in H^{1}(M ; \mathbb{Z} / 2)$ and $\beta \in H^{2}(M ; \mathbb{Z} / 2)$. The non-trivial cup products are:

(i) $\theta_{l} \cdot \varphi_{l}=\gamma$.

(ii) If $A e+C \equiv 0(\bmod 2)$, then

$$
\begin{aligned}
\alpha^{2} & =\left[q+\frac{1}{2}(A e+C)\right] \beta, & \theta_{l}^{2} & =\beta, \\
\alpha^{3} & =\left[q+\frac{1}{2}(A e+C)\right] \gamma, & \alpha \cdot \beta & =\gamma
\end{aligned}
$$

4. Applications to degree one maps and Lusternik-Schnirelmann category. By [7] Theorem 2.2, for given $p$, a degree one map from an orientable 3-manifold $M$ onto a lens space $L(p, q)$ exists, for some $q$, if and only if there exists an element $x \in H^{1}(M ; \mathbb{Z} / p)$ such that the linking number $x \odot x=\left\langle x \cdot B_{p}(x),[M]\right\rangle \neq 0$. The following result determines 
all possible non-zero values for $x \cdot B_{p}(x)$ in any orientable Seifert manifold $M$, and is a direct corollary of the previous results. Observe that when $p=2, L(2, q)=L(2,1)=\mathbb{R} \mathrm{P}^{3}$ and $x \cdot B_{p}(x)=x^{3}$ so that the description of degree one maps given in Corollary 4.2 agrees with the description in Theorem 4.14 [2], which is restated in Corollary 4.3. It is worth noting that in the cases where a degree one map $M \rightarrow L(p, q)$ exists, the value of $q$ is not arbitrary. By [7] Theorem 2.2, if the linking number $x \odot x=[r / p] \in \mathbb{Q} / \mathbb{Z}$, then $q r \equiv 1$ $(\bmod p)$.

Theorem 4.1. For any prime $p$, and any orientable Seifert manifold $M$ let $x$ be an element of $H^{1}(M ; \mathbb{Z} / p)$. Then all possible non-zero values of $x \cdot B_{p}(x)$ are given by:

(i) For the Seifert manifold $M \cong\left(O, o, g \mid e:\left(a_{1}, b_{1}\right), \ldots,\left(a_{m}, b_{m}\right)\right)$, when $p \geq 2$ and $n_{p} \geq 2$,

$$
\alpha_{i} \cdot B_{p}\left(\alpha_{i}\right)=\left(a_{i}^{\prime} c_{i}+a_{1}^{\prime} c_{1}\right) \gamma, 2 \leq i \leq n_{p} .
$$

(ii) For $M \cong\left(O, o ; g \mid e:\left(a_{1}, b_{1}\right), \ldots,\left(a_{m}, b_{m}\right)\right)$, with $n_{p}=0$ and $A e+C \equiv 0(\bmod p)$,

$$
\alpha \cdot B_{p}(\alpha)=A^{-1}\left(\sum_{i=1}^{r} b_{i}^{\prime} A_{i}+\left(\frac{A e+C}{p}\right)\right) \gamma .
$$

(iii) For $M \cong\left(O, n ; g \mid e:\left(a_{1}, b_{1}\right), \ldots,\left(a_{m}, b_{m}\right)\right)$, when $p>2$ and $n_{p}>0$,

$$
\begin{aligned}
\alpha_{i} \cdot B_{p}\left(\alpha_{i}\right) & =\left(a_{i}^{\prime} c_{i}+a_{1}^{\prime} c_{1}\right) \gamma, 2 \leq i \leq n_{p}, \\
\theta_{l} \cdot B_{p}\left(\theta_{l}\right) & =-4 a_{1}^{\prime} c_{1} \gamma .
\end{aligned}
$$

(iv) For $M \cong\left(O, n ; g \mid e:\left(a_{1}, b_{1}\right), \ldots,\left(a_{m}, b_{m}\right)\right)$, when $p=2, n_{2}=0$, and $A e+C \equiv 0$ $(\bmod 2)$

$$
\alpha^{3}=\alpha \cdot B_{2}(\alpha)=\left(q+\frac{A e+C}{2}\right) \gamma,
$$

where $q$ is defined in Theorem $3.3(i)$.

Corollary 4.2. Let $p>2$ be a fixed prime. Then any orientable Seifert manifold $M$ admits a degree one map to $L(p, q)$, for some $q$, precisely in the following cases.

(i) $M=\left(O, o ; g \mid e:\left(a_{1}, b_{1}\right), \ldots,\left(a_{m}, b_{m}\right)\right), n_{p} \geq 3$, and $a_{1}, \ldots, a_{n_{p}}$ not all divisible by $p^{2}$

(ii) $M$ as in (a) with $n_{p}=2$, and $p^{-1}\left(a_{1} c_{1}+a_{2} c_{2}\right) \not \equiv 0(\bmod p)$,

(iii) $M$ as in (a) with $n_{p}=0, A e+C \equiv 0(\bmod p)$, and $p^{-1}\left(\sum_{i=1}^{r} b_{i} A_{i}+A e+C\right) \not \equiv 0$ $(\bmod p)$,

(iv) $M=\left(O, n ; g \mid e:\left(a_{1}, b_{1}\right), \ldots\left(a_{m}, b_{m}\right)\right), n_{p} \geq 1$, and $a_{1}, \ldots, a_{n_{p}}$ not all divisible by $p^{2}$.

Outline of proof. The proofs of (ii), (iii), and (iv) are self evident from Theorem 4.1. To prove (i), suppose there is no degree one map $M \rightarrow L(p, q)$, that is, $x \cdot B_{p}(x)=0$, for all $x \in H^{1}(X ; \mathbb{Z} / p)$. First consider $x=\alpha_{i}$ for $2 \leq i \leq p$ and secondly let $x=\alpha_{j}-\alpha_{i}$ for $2 \leq i<j \leq n_{p}$ (this choice is possible because $n_{p} \geq 3$ ). A short calculation then shows that $0=a_{i}^{\prime} c_{i} \in \mathbb{Z} / p$, for $1 \leq i \leq n_{p}$ and this implies that $a_{i}^{\prime} \equiv 0(\bmod p)\left(\right.$ since $c_{i} \not \equiv 0$ $(\bmod p))$. Thus $a_{i} \equiv 0\left(\bmod p^{2}\right)$. Taking the contrapositive gives the result. 
Corollary 4.3. For $p=2$ and $M$ an orientable Seifert manifold as above (of type $(\mathrm{O}, \mathrm{o})$ or $(\mathrm{O}, \mathrm{n})), M$ admits a degree one map to $R P^{3}$ in precisely the following cases.

(i) $n_{2} \geq 2$, and $\frac{\left(a_{i}+a_{j}\right)}{2} \equiv 1(\bmod 2)$ for some $i, j, 1 \leq i, j \leq m$,

(ii) $n_{2}=0, A e+C \equiv 0(\bmod 2)$, and $q+\frac{(A e+C)}{2} \equiv 1(\bmod 2)$.

This corollary is also of interest in relativity theory (cf. [19]).

Consider the set, $\mathcal{L}$, of all open coverings of a space $X$ in which each open set of the covering $C \in \mathcal{L}$ is contractible in $X$. Let $|C|$ be the cardinality of the covering $C \in \mathcal{L}$. The Lusternik-Schnirelmann category of $X$ is defined to be $\min \{|C|: C \in \mathcal{L}\}$. Let cat $(X)$ denote the normalized Lusternik-Schnirelmann category, which is defined to be one less than the category defined above. Thus if $X$ is contractible, cat $(X)=0$. For a comprehensive review of this subject, see James' survey article [10]. It should be remarked though, that James does not use the normalized version of category. For further references on this subject see Fox [6], Eilenberg and Ganea [4], and Rudyak [15]. A second application of the results presented in $\S 3$ determines the normalized Lusternik-Schnirelmann category of a class of Seifert manifolds, by finding the cup product length of the Seifert manifold $M$ (that is, the longest sequence of elements $u_{1}, \ldots, u_{k}$ in cohomology of positive degree such that $u_{1} \smile u_{2} \smile \ldots \smile u_{k} \neq 0$ ).

REMARK 4.4.

(i) Let $X$ be a $C W$-complex of dimension $k$. Then cat $(X) \leq k$ (cf. [6], [10]).

(ii) Let $X$ be a topological space with cat $(X) \leq k$ and let $u_{1}, \ldots, u_{k+1}$ be any $k+1$ cohomology classes of $X$. Then $u_{1} \smile u_{2} \smile \ldots \smile u_{k+1}=0$ (cf. [21] Lemma 2.2 and $[20]$ p. 279).

(iii) $\operatorname{cat}(X \times Y) \leq \operatorname{cat}(X)+\operatorname{cat}(Y)$ (cf. [10]).

It follows immediately from Remark 4.4 (ii) that if $X$ has cup product length $k$, then cat $(X) \geq k$. Henceforth the cup product length of a space $X$ will be denoted by $\operatorname{cup}(X)$.

The next theorem is obtained by simply applying Remark 4.4 to each case. The notation is that used in $\S 3$.

Theorem 4.5. (i) Let $M \cong\left(O, o ; g \mid e:\left(a_{1}, b_{1}\right), \ldots,\left(a_{m}, b_{m}\right)\right)$. If $n_{2} \geq 2$ and $a_{i} \equiv 2$ $(\bmod 4)$ for some $i, 1 \leq i \leq n_{2}$, or if $n_{p}=0$ and $A e+C \equiv 0(\bmod p)$, then cat $(M)=3$.

(ii) Let $M \cong\left(O, n ; g \mid e:\left(a_{1}, b_{1}\right), \ldots,\left(a_{m}, b_{m}\right)\right)$. If $n_{2} \geq 2$ and $a_{i} \equiv 2(\bmod 4)$ for some $i, 1 \leq i \leq n$, or if $n_{2}=0$ and $A e+C \equiv 0(\bmod 2)$, then $\operatorname{cat}(M)=3$.

Proof. (i) By Theorem 3.1 (i) $\operatorname{cup}(M)=3$ when $a_{i} \equiv 2(\bmod 4)$, for some $i$, $1 \leq i \leq n_{2}$. Furthermore, Theorem 3.3 (ii) shows that when $n_{p}=0$ and $A e+C \equiv 0$ $(\bmod p), \theta_{l} \cdot \theta_{l}^{\prime}=\beta$ and $\alpha \cdot \beta=\gamma$. Thus $\operatorname{cup}(M)=3$ in this case as well.

(ii) As in (i), Theorem 3.4 (i) shows that $\operatorname{cup}(M)=3$ when $a_{i} \equiv 2(\bmod 4)$, for some $i, 1 \leq i \leq n_{2}$. Finally when $n_{2}=0$ and $A e+C \equiv 0(\bmod 2)$, it follows from Theorem 3.7 (ii) that $\theta_{l}^{2}=\beta$ and $\alpha \cdot \beta=\gamma$. So again $\operatorname{cup}(M)=3$.

It should be noted that Theorem 4.5 can also be deduced from the results of Eilenberg and Ganea [4], who considered Eilenberg-MacLane spaces in general. 
Recall that for every finite CW-complex and every integer $m>0$ the Ganea conjecture states that cat $\left(X \times S^{m}\right)=\operatorname{cat}(X)+1$. This conjecture has recently been disproved in general by Iwase [9]. However, the next result describes a new class of spaces for which the Ganea conjecture is true.

THEOREM 4.6. Let $M$ be an orientable Seifert manifold satisfying either of the conditions in Theorem $4.5(i)$ or $(i i)$. Then,

$$
\text { cat }\left(M \times S^{m_{1}} \times \ldots \times S^{m_{k}}\right)=\operatorname{cat}(M)+k=3+k .
$$

Proof. By Remark 4.4 (i) cat $(M) \leq \operatorname{dim}(M)=3$. By Theorem 4.5 and Remark 4.4 (ii) $\operatorname{cup}(M) \geq \operatorname{cat}(M)=3$. Thus $\operatorname{cup}(M)=\operatorname{cat}(M)$ (for the appropriate coefficients described in Theorem 4.5). Furthermore observe that for any coefficients, cup $\left(S^{m_{1}} \times \ldots \times S^{m_{k}}\right)=k$. Thus (for the appropriate coefficients) the Künneth formula and Remark 4.4 (ii), (iii) give:

$$
\begin{aligned}
3+k & \leq \operatorname{cup}\left(M \times S^{m_{1}} \times \ldots \times S^{m_{k}}\right) \\
& \leq \operatorname{cat}\left(M \times S^{m_{1}} \times \ldots \times S^{m_{k}}\right) \\
& \leq \operatorname{cat}(M)+\operatorname{cat}\left(S^{m_{1}} \times \ldots \times S^{m_{k}}\right) \leq 3+k .
\end{aligned}
$$

Therefore cat $\left(M \times S^{m_{1}} \times \ldots \times S^{m_{k}}\right)=3+k$.

Observe that the proof of 4.6 is valid for any $n$-manifold $M^{n}$ such that cup $(M)=n$.

If $M$ is a connected PL manifold that satisfies $\operatorname{dim} M \leq 2$ cat $M-3$ then by [15] Theorem G, cat $\left(M \times S^{m_{1}} \times \ldots \times S^{m_{k}}\right)=\operatorname{cat}(M)+k$. Since any 3 -manifold is a PL manifold (cf. [8]), Theorem 4.6 can also be deduced from [15] Theorem G and Theorem 4.5 , by noting that $\operatorname{dim} M \leq 2$ cat $M-3=3$.

Appendix. Consider the equivariant chain complex $\mathcal{C}:=\left\{C_{i}\right\}$ of $\S 2$. For any generator $\alpha$ of $C_{i}$, let $\hat{\alpha}$ denote the dual generator of $\operatorname{Hom}_{R}\left(C_{i} ; \mathbb{Z} / p\right)$; that is, $\hat{\alpha}(\alpha)=1, \hat{\alpha}(\beta)=0$ for any other generator $\beta$ of $C_{i}$, for $i=0,1,2,3$.

Theorem A.1. Let $M=\left(O, o ; g \mid e:\left(a_{1}, b_{1}\right), \ldots,\left(a_{m}, b_{m}\right)\right)$. Then its $\mathbb{Z} / p$ cohomology groups have the following generators:

CASE $(1):$ Let $a_{1}, \ldots, a_{n_{p}} \equiv 0(\bmod p), 1 \leq n_{p} \leq m$, and $a_{n_{p}+1}, \ldots, a_{m} \equiv 1(\bmod p)$. Then

$$
\begin{cases}1=\left[\sum_{j=0}^{m} \hat{\sigma}_{j}^{0}\right], & \text { degree } 0, \\ \alpha_{j}:=\left[\hat{\rho}_{j}^{1}-\hat{\rho}_{1}^{1}\right], \theta_{k}:=\left[\hat{\nu}_{k}^{1}\right], \theta_{k}^{\prime}:=\left[\hat{\omega}_{k}^{1}\right], 2 \leq j \leq n_{p}, 1 \leq k \leq g, & \text { degree } 1, \\ \beta_{j}:=\left[\hat{\sigma}_{j}^{2}\right]=\left[b_{j} \hat{\mu}_{j}^{2}\right], \varphi_{k}:=\left[\hat{\nu}_{k}^{2}\right], \varphi_{k}^{\prime}:=\left[\hat{\omega}_{k}^{2}\right], 2 \leq j \leq n_{p}, 1 \leq k \leq g, & \text { degree } 2, \\ \gamma:=\left[\hat{\delta}^{3}\right]=\left[-\hat{\sigma}_{0}^{3}\right]=\ldots=\left[-\hat{\sigma}_{m}^{3}\right], & \text { degree } 3 .\end{cases}
$$

CASE $(2):$ Assume that $n_{p}=0, b_{i} \equiv 0(\bmod p)$ for $1 \leq i \leq r, b_{i} \not \equiv 0(\bmod p)$ for $r+1 \leq i \leq m$. Then 


$$
\begin{cases}1=\left[\sum_{j=0}^{m} \hat{\sigma}_{j}^{0}\right], & \text { degree } 0, \\ \theta_{k}:=\left[\hat{\nu}_{k}^{1}\right], \theta_{k}^{\prime}:=\left[\hat{\omega}_{k}^{1}\right], 1 \leq k \leq g, & \text { degree } 1, \\ \varphi_{k}:=\left[\hat{\nu}_{k}^{2}\right], \varphi_{k}^{\prime}:=\left[\hat{\omega}_{k}^{2}\right], 1 \leq k \leq g, & \text { degree } 2, \\ \alpha:=\left[\sum_{j=0}^{m} \hat{\eta}_{j}^{1}-\sum_{j=r+1}^{m} b_{j} a_{j}^{-1} \hat{\rho}_{j}^{1}-e \hat{\rho}_{0}^{1}\right], \theta_{k}, \theta_{k}^{\prime}, 1 \leq k \leq g, \text { Ae }+C \equiv 0(\bmod p), & \text { degree } 1, \\ \beta:=\left[\hat{\delta}^{2}\right]=\left[\hat{\mu}_{0}^{2}\right]=\ldots=\left[\hat{\mu}_{m}^{2}\right], \varphi_{k}, \varphi_{k}^{\prime}, 1 \leq k \leq g, \text { Ae }+C \equiv 0(\bmod p), & \text { degree } 2, \\ \gamma:=\left[\hat{\delta}^{3}\right]=\left[-\hat{\sigma}_{0}^{3}\right]=\ldots=\left[-\hat{\sigma}_{m}^{3}\right], & \text { degree } 3 .\end{cases}
$$

TheOrem A.2. Let $M=\left(O, n ; g \mid e:\left(a_{1}, b_{1}\right), \ldots,\left(a_{m}, b_{m}\right)\right)$. Then the $\mathbb{Z} / p$ cohomology groups have the same generators that are given in Theorem A.1, in both cases, except that the classes $\theta_{k}^{\prime}$ and $\phi_{k}^{\prime}$ are deleted.

\section{References}

[1] J. Bryden, C. Hayat-Legrand, H. Zieschang and P. Zvengrowski, L'anneau de cohomologie d'une variété de Seifert, C. R. Acad. Sci. Paris 324, Sér. I (1997), 323-326.

[2] J. Bryden, C. Hayat-Legrand, H. Zieschang and P. Zvengrowski, The cohomology ring of a class of Seifert manifolds, Top. and its Appl., to appear.

[3] J. Bryden and P. Zvengrowski, The cohomology ring of the orientable Seifert manifolds $I I$, preprint.

[4] S. Eilenberg and T. Ganea, On the Lusternik-Schnirelmann category of abstract groups, Ann. of Math. 65 (1957), 517-518.

[5] R. H. Fox, Free differential calculus. I. Derivations in the free group ring, Ann. of Math. 57 (1953), 547-560.

[6] R. H. Fox, On the Lusternik-Schnirelmann category, Ann. of Math. 42 (1941), 333-370.

[7] C. Hayat-Legrand, S. Wang and H. Zieschang, Degree-one maps onto lens spaces, Pac. J. Math. 176 (1996), 19-32.

[8] J. Hempel, 3-Manifolds, Annals of Math. Studies, vol. 86, Princeton University Press, Princeton, New Jersey 1976, 115-135.

[9] N. Iwase, Ganea's conjecture on Lusternik-Schnirelmann category, preprint.

[10] I. M. James, On category, in the sense of Lusternik-Schnirelmann, Topology 17 (1978), 331-348.

[11] S. MacLane, Homology, Springer-Verlag, Berlin, 1963.

[12] J. M. Montesinos, Classical Tesselations and Three-Manifolds, Springer-Verlag, Berlin, 1987.

[13] P. Orlik, Seifert Manifolds, Lecture Notes in Math. 291, Springer-Verlag, Berlin, 1972.

[14] K. Reidemeister, Homotopieringe und Linsenräume, Abh. Math. Sem. Univ. Hamburg 11 (1935), 102-109.

[15] Y. B. Rudyak, On category weight and its applications, preprint.

[16] P. Scott, The geometries of 3-manifolds, Bull. London Math. Soc. 15 No. 56 (1983), 401-487. 
[17] H. Seifert, Topologie dreidimensionaler gefaserter Räume, Acta Math. 60 (1932), 147238.

[18] H. Seifert and W. Threlfall, A Textbook of Topology, Academic Press, 1980.

[19] A. R. Shastri, J. G. Williams and P. Zvengrowski, Kinks in general relativity, International Journal of Theoretical Physics 19 (1980), 1-23.

[20] E. H. Spanier, Algebraic Topology, McGraw-Hill, New York, 1966.

[21] N. Steenrod and D. B. A. Epstein, Cohomology Operations, The University of Princeton Press, Princeton, N.J., 1962. 